\title{
Quantization of Drinfeld zastava in type C
}

\author{
Michael Finkelberg and Leonid Rybnikov
}

To Vladimir Drinfeld on his 60th birthday

\begin{abstract}
A Drinfeld zastava is a certain closure of the moduli space of maps from the projective line to the Kashiwara flag scheme of an affine Lie algebra $\hat{\mathfrak{g}}$. In case $\mathfrak{g}$ is the symplectic Lie algebra $\mathfrak{s p}_{N}$, we introduce an affine, reduced, irreducible, normal quiver variety $Z$ which maps to the zastava space isomorphically in characteristic 0. The natural Poisson structure on the zastava space $Z$ can be described in terms of the Hamiltonian reduction of a certain Poisson subvariety of the dual space of a (nonsemisimple) Lie algebra. The quantum Hamiltonian reduction of the corresponding quotient of its universal enveloping algebra produces a quantization $Y$ of the coordinate ring of $Z$. The same quantization was obtained in the finite (as opposed to the affine) case generically in the work of Gerasimov-Kharchev-Lebedev-Oblezin (2005). We prove that $Y$ is a quotient of the affine Borel Yangian. The analogous results for $\mathfrak{g}=\mathfrak{s l}_{N}$ were obtained in our previous work.
\end{abstract}

\section{Introduction}

1.1. This note is a continuation of [FR14], where we have studied the Drinfeld zastava spaces $Z \stackrel{d}{d}\left(\widehat{\mathfrak{s l}}_{N}\right)$ from the viewpoint of invariant theory. Given a collection of complex vector spaces $\left(V_{l}\right)_{l \in \mathbb{Z} / N \mathbb{Z}}$ with dimension vector $\underline{\operatorname{dim}}\left(V_{l}\right)_{l \in \mathbb{Z} / N \mathbb{Z}}=\left(d_{l}\right)_{l \in \mathbb{Z} / N \mathbb{Z}}=\underline{d}$ along with a collection of vector spaces $\left(W_{l}\right)_{l \in \mathbb{Z} / N \mathbb{Z}}$ with dimension vector $\underline{\operatorname{dim}}\left(W_{l}\right)_{l \in \mathbb{Z} / N \mathbb{Z}}=(1, \ldots, 1)$, we consider the space $M_{\underline{d}}=\left\{\left(A_{l}, B_{l}, p_{l}, q_{l}\right)_{l \in \mathbb{Z} / N \mathbb{Z}}\right\}=$

$$
\bigoplus_{l \in \mathbb{Z} / N \mathbb{Z}} \operatorname{End}\left(V_{l}\right) \oplus \bigoplus_{l \in \mathbb{Z} / N \mathbb{Z}} \operatorname{Hom}\left(V_{l}, V_{l+1}\right) \oplus \bigoplus_{l \in \mathbb{Z} / N \mathbb{Z}} \operatorname{Hom}\left(W_{l-1}, V_{l}\right) \oplus \bigoplus_{l \in \mathbb{Z} / N \mathbb{Z}} \operatorname{Hom}\left(V_{l}, W_{l}\right)
$$

of representations of the following chainsaw quiver:

Received 26 Juli 2013, accepted in final form 24 September 2013.

2010 Mathematics Subject Classification 19E08, (22E65, 37K10)

Keywords: chainsaw quiver, quadratic spaces, affine Yangian, hamiltonian reduction, quantization.

This journal is (c) Foundation Compositio Mathematica 2014. This article is distributed with Open Access under the terms of the Creative Commons Attribution Non-Commercial License, which permits non-commercial reuse, distribution, and reproduction in any medium, provided that the original work is properly cited. For commercial re-use, please contact the Foundation Compositio Mathematica.

Both authors were partially supported by the RFBR grants 12-01-00944, 12-01-33101, 13-01-12401/13, the National Research University Higher School of Economics' Academic Fund award No.12-09-0062 and the AG Laboratory HSE, RF government grant, ag. 11.G34.31.0023. This study comprises research findings from the "Representation Theory in Geometry and in Mathematical Physics" carried out within The National Research University Higher School of Economics' Academic Fund Program in 2012, grant No 12-05-0014. L.R. was supported by the RFBR grant 11-01-93105-CNRSL-a. This study was carried out within The National Research University Higher School of Economics Academic Fund Program in 2013-2014, research grant No. 12-01-0065. 


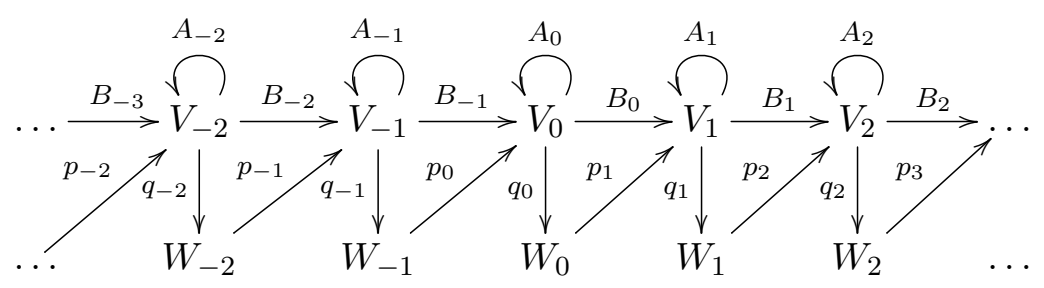

Furthermore, we consider the closed subscheme $\mathrm{M}_{\underline{d}} \subset M_{\underline{d}}$ cut out by the equations $A_{l+1} B_{l}-$ $B_{l} A_{l}+p_{l+1} q_{l}=0$ for all $l$, and two open subschemes $M_{d}^{s} \subset M_{\underline{d}}$ (resp. $M_{d}^{c} \subset M_{\underline{d}}$ ) formed by

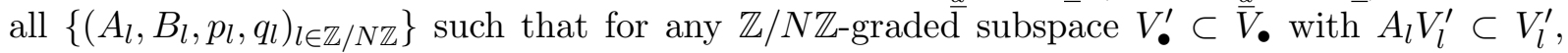
and $B_{l} V_{l}^{\prime} \subset V_{l+1}^{\prime}$ for all $l$, if $p_{l}\left(W_{l-1}\right) \subset V_{l}^{\prime}$ for all $l$, then $V_{\bullet}^{\prime}=V_{\bullet}$ (resp. if $V_{l}^{\prime} \subset \operatorname{Ker} q_{l}$ for all $l$, then $\left.V_{\bullet}^{\prime}=0\right)$. The group $G\left(V_{\bullet}\right)=\prod_{l \in \mathbb{Z} / N \mathbb{Z}} G L\left(V_{l}\right)$ acts naturally on $M_{\underline{d}}$ preserving the subschemes $\mathrm{M}_{\underline{d}}, M_{\underline{d}}^{s}, M_{\underline{d}}^{c}$.

According to [FR14], [BF11], the action of $G\left(V_{\bullet}\right)$ on $\mathrm{M}_{\underline{d}} \cap M_{d}^{s} \cap M_{d}^{c}$ is free, and the quotient $\left(\mathrm{M}_{\underline{d}} \cap M_{\underline{d}}^{s} \cap M_{\underline{d}}^{c}\right) / G\left(V_{\bullet}\right)$ is naturally isomorphic to the moduli space $\stackrel{\circ}{Z}^{-}$of based maps of degree $\underline{d}$ from the projective line to the Kashiwara flag scheme of the affine Lie algebra $\widehat{\mathfrak{s l}}_{N}$. Moreover, the categorical quotient $\mathrm{M}_{d} / / G\left(V_{\bullet}\right)$ is naturally isomorphic to the Drinfeld zastava closure $Z \underline{d}\left(\widehat{\mathfrak{s l}}_{N}\right) \supset \stackrel{\circ}{Z^{d}}$. Furthermore, the scheme $\mathrm{M}_{\underline{d}} / / G\left(V_{\bullet}\right) \simeq Z^{\underline{d}}\left(\widehat{\mathfrak{s l}}_{N}\right)$ is reduced, irreducible and normal. One of the crucial points in proving this consists in checking that $\left(\mathrm{M}_{\underline{d}} \cap M_{\underline{d}}^{s} \cap M_{\underline{d}}^{c}\right) \subset \mathrm{M}_{\underline{d}}$ is dense, while $\mathrm{M}_{\underline{d}} \subset M_{\underline{d}}$ is a complete intersection.

1.2. One of the goals of this note is to extend the above results to the case of zastava spaces for the affine symplectic Lie algebra $\widehat{\mathfrak{s p}}_{N}$ (in case $N$ is even). We prove that any zastava scheme for $\widehat{\mathfrak{s p}}_{N}$ is reduced, irreducible and normal. Note that these properties of zastava schemes were established in [BF11] for all finite dimensional simple Lie algebras.

To this end we again invoke invariant theory. Following [BS00], we equip $W:=\bigoplus_{l \in \mathbb{Z} / N \mathbb{Z}} W_{l}$ with a symplectic form such that $W_{l}$ and $W_{k}$ are orthogonal unless $l+k=N-1$. We equip $V:=\bigoplus_{l \in \mathbb{Z} / N \mathbb{Z}} V_{l}$ with a nondegenerate symmetric bilinear form such that $V_{l}$ and $V_{k}$ are orthogonal unless $l+k=0$. In particular, we must have $d_{-l}=d_{l}$ for all $l$, so that the collection $\underline{d}$ is encoded by $\bar{d}:=\left(d_{0}, d_{1}, \ldots, d_{N / 2}\right)$. We denote by $O\left(V_{\bullet}\right)$ the Levi subgroup of the orthogonal group $O(V)$ preserving the decomposition $V:=\bigoplus_{l \in \mathbb{Z} / N \mathbb{Z}} V_{l}$. We consider the space $M_{\underline{d}}^{-1} \subset M_{\underline{d}}$ of representations of the quadratic chainsaw quiver formed by all the selfadjoint collections $A_{l}^{*}=A_{l}, B_{l}^{*}=B_{-l-1}, p_{l}^{*}=q_{-l}$. We denote by $\mathrm{M}_{\underline{d}}^{-1} \subset M_{\underline{d}}^{-1}$ the scheme-theoretic intersection $\mathrm{M}_{\underline{d}} \cap M_{\underline{d}}^{-1}$.

We prove that $\mathrm{M}_{\underline{d}}^{-1} \cap M_{\underline{d}}^{s}=\mathrm{M}_{\underline{d}}^{-1} \cap M_{\underline{d}}^{c} \subset \mathrm{M}_{\underline{d}}^{-1}$ is dense, while $\mathrm{M}_{\underline{d}}^{-1} \subset M_{\underline{d}}^{-1}$ is a complete intersection. We deduce that the categorical quotient $\mathrm{M}_{d}^{-1} / / O\left(V_{\bullet}\right)$ is reduced, irreducible and normal. Furthermore, we prove that the action of $O\left(V_{\bullet}\right)$ on $\mathrm{M}_{\underline{d}}^{-1} \cap M_{\underline{d}}^{s}=\mathrm{M}_{\underline{d}}^{-1} \cap M_{\underline{d}}^{c}$ is free and that the quotient $\left(\mathrm{M}_{\underline{\underline{d}}}^{-1} \cap M_{\underline{d}}^{s}\right) / O\left(V_{\bullet}\right)$ is naturally isomorphic to the moduli space $Z^{\bar{d}}\left(\widehat{\mathfrak{s p}}_{N}\right)$ of based maps of degree $\overline{\bar{d}}$ from the projective line to the Kashiwara flag scheme of the affine Lie algebra $\widehat{\mathfrak{s p}}_{N}$. Moreover, the categorical quotient $\mathrm{M}_{\underline{d}}^{-1} / / G\left(V_{\bullet}\right)$ is naturally isomorphic to the Drinfeld zastava closure $Z^{\bar{d}}\left(\widehat{\mathfrak{s p}}_{N}\right) \supset Z^{\bar{d}}\left(\widehat{\mathfrak{s p}}_{N}\right)$. 


\section{Michael Finkelberg and Leonid Rybnikov}

1.3. Quite naturally, we would like to extend the above results to the case of the affine orthogonal Lie algebra $\widehat{\mathfrak{s o}}_{N}$. To this end we change the parities of the bilinear forms in 1.2. That is, we equip $W$ with a nondegenerate symmetric bilinear form, and we equip $V$ with a symplectic form. The corresponding space of representations of the quadratic chainsaw quiver is denoted by $M_{d}^{1}$, and the corresponding Levi subgroup of $S p(V)$ is denoted by $S p\left(V_{\bullet}\right)$. It is still true that the action of $S p\left(V_{\bullet}\right)$ on $\mathrm{M}_{\underline{d}}^{1} \cap M_{\underline{d}}^{s}=\mathrm{M}_{\underline{d}}^{1} \cap M_{\underline{d}}^{c}$ is free, and the quotient $\left(\mathrm{M}_{\underline{d}}^{1} \cap M_{\underline{d}}^{s}\right) / S p\left(V_{\bullet}\right)$ is naturally isomorphic to $\stackrel{\circ}{Z}^{\bar{d}}\left(\widehat{\mathfrak{s o}}_{N}\right)$.

However, we encounter the following mysterious obstacle: $\mathrm{M}_{\underline{d}}^{1}$ is not irreducible in general, and $\mathrm{M}_{\underline{d}}^{1} \cap M_{\underline{d}}^{s}=\mathrm{M}_{\underline{d}}^{1} \cap M_{\underline{d}}^{c}$ is only dense in one of its irreducible components. For this reason the categorical quotient $\mathrm{M}_{\underline{d}}^{1} / / S p\left(V_{\bullet}\right)$ is not isomorphic to the zastava space $Z^{\bar{d}}\left(\widehat{\mathfrak{s o}}_{N}\right)$ in general. The simplest example occurs when $\bar{d}$ is the affine simple coroot of $\widehat{\mathfrak{s o}}_{N}$, that is, $\underline{d}=(\ldots, 0,1,2,1,0, \ldots)$.

1.4. Following [FR14], we describe the natural Poisson structure on $Z^{\bar{d}}\left(\widehat{\mathfrak{s p}}_{N}\right)$ in quiver terms. It is obtained by the Hamiltonian reduction of a Poisson subvariety of the dual vector space of a (non-semisimple) Lie algebra $\mathfrak{a}_{d}^{-1}$ with its Lie-Kirillov-Kostant bracket. Now the ring of functions $\mathbb{C}\left[Z^{\bar{d}}\left(\widehat{\mathfrak{s p}}_{N}\right)\right]$ admits a natural quantization $y_{\bar{d}}^{-1}$ as the quantum Hamiltonian reduction of a quotient algebra of the universal enveloping algebra $U\left(\mathfrak{a}_{\underline{d}}^{-1}\right)$. The algebra $y_{\bar{d}}^{-1}$ admits a homomorphism from the Borel subalgebra $y^{-1}$ of the Yangian of type $C$ in the case of a finite zastava space. We prove that this homomorphism is surjective. In the affine situation, there is an affine analog $\widehat{y}^{-1}$ of $y^{-1}$ (it is no longer a subalgebra in the Yangian of $\widehat{\mathfrak{s p}}_{N}$ ), and we define it explicitly by generators and relations. We prove that there is a surjective homomorphism $\widehat{y}^{-1} \rightarrow y_{\bar{d}}^{-1}$. Moreover, we write down certain elements in the kernel of this homomorphism and conjecture that they generate the kernel (as a two-sided ideal). These elements are similar to the generators of the kernel of the Kamnitzer-Webster-Weekes-Yacobi homomorphism from the shifted Yangian to the quantization of the transversal slices in the affine Grassmannian. In fact, as explained in [KWWY12], $y_{\bar{d}}^{-1}$ as a filtered algebra is the limit of a sequence of quantum coordinate rings of transversal slices.

\section{A quiver approach to Drinfeld zastava for symplectic groups}

\subsection{Quadratic spaces}

We will recall the convenient terminology introduced in [KP82]. Let $U$ be an $N$-dimensional complex vector space equipped with a nondegenerate bilinear form $($,$) such that (u, v)=\varepsilon(v, u)$. It will be called a quadratic space of type $\varepsilon$ (shortened to orthogonal space if $\varepsilon=1$ and symplectic space if $\varepsilon=-1)$. We denote by $G_{\varepsilon}(U)$ the subgroup of $G L(U)$ leaving the form invariant. So we have $G_{\varepsilon}(U)=O(N)$ or $S p(N)$ according to whether $\varepsilon=1$ or $\varepsilon=-1$.

Let $A \mapsto A^{*}, \operatorname{End}(U) \rightarrow \operatorname{End}(U)$ be the canonical involution associated with the form, that is, $(A u, v)=\left(u, A^{*} v\right)$ for any $u, v \in U$. More generally, for a linear operator $B \in \operatorname{Hom}\left(U,{ }^{\prime} U\right)$ we denote by $B^{*}$ the adjoint (or transposed) operator $B^{*} \in \operatorname{Hom}\left({ }^{\prime} U^{*}, U^{*}\right)$.

We choose a basis $w_{0}, \ldots, w_{N-1}$ in a quadratic space $W$ of type $\varepsilon=-1$ such that for $0 \leqslant l<N / 2$ we have $\left(w_{l}, w_{m}\right)=\delta_{m, N-1-l}$ (note that $N$ is necessarily even). The linear span of $w_{l}$ will be denoted by $W_{l} \cong \mathbb{C}$. We will often parametrize the base vectors by the elements of $\mathbb{Z} / N \mathbb{Z}$. We define $I:=\{0,1, \ldots, N / 2\} \subset\{0, \ldots, N-1\}=\mathbb{Z} / N \mathbb{Z}$. We set $I=I_{0} \sqcup I_{1}$ where $I_{0}=\left\{1, \ldots, \frac{1}{2} N-1\right\}, I_{1}=\left\{0, \frac{1}{2} N\right\}$. 


\section{Quantization of Drinfeld Zastava in type C}

We choose another quadratic space $V$ of type $-\varepsilon=1$ decomposed into a direct sum $V=\bigoplus_{l \in \mathbb{Z} / N \mathbb{Z}} V_{l}$ such that $V_{l}$ is orthogonal to $V_{m}$ unless $l+m=0 \in \mathbb{Z} / N \mathbb{Z}$. We denote the dimension of $V_{l}$ by $d_{l}$. We set $\underline{d}:=\left(d_{l}\right)_{l \in I}$.

We denote by $G_{-\varepsilon}\left(V_{\bullet}\right)$ the Levi subgroup of $G_{-\varepsilon}(V)$ preserving the decomposition $V=\bigoplus_{l \in \mathbb{Z} / N \mathbb{Z}} V_{l}$. It is isomorphic to $O\left(V_{0}\right) \times O\left(V_{N / 2}\right) \times \prod_{0<l<N / 2} G L\left(V_{l}\right)$.

\subsection{Quadratic chainsaw quivers}

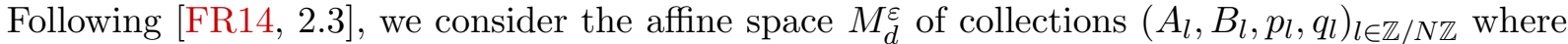
$A_{l} \in \operatorname{End}\left(V_{l}\right), B_{l} \in \operatorname{Hom}\left(V_{l}, V_{l+1}\right), p_{l} \in \operatorname{Hom}\left(W_{l-1}, \bar{V}_{l}\right), q_{l} \in \operatorname{Hom}\left(V_{l}, W_{l}\right)$ satisfy the following selfadjointness conditions: $A_{l}^{*}=A_{-l}, B_{l}^{*}=B_{-l-1}, p_{l}^{*}=q_{-l}$. Here we view $p_{l}$ (resp. $q_{l}$ ) as a vector (resp. covector) of $V_{l}$ using the identification of all $W_{m}$ with $\mathbb{C}$.

Following loc. cit., we consider the subscheme $\mathrm{M}_{d}^{\varepsilon} \subset M_{d}^{\varepsilon}$ parametrizing the $\underline{d}$-dimensional representations of the chainsaw quiver with bilinear form (or the quadratic chainsaw quiver for short), cut out by the equations $A_{l+1} B_{l}-B_{l} A_{l}+p_{l+1} q_{l}=0$ for all $l$.

Clearly, $\mathrm{M}_{d}^{\varepsilon}$ is acted upon by the Levi subgroup $G_{-\varepsilon}\left(V_{\bullet}\right)$. We denote by $\mathfrak{Z}_{d}^{\varepsilon}$ the categorical quotient $\mathrm{M}_{d}^{\varepsilon} / / \bar{G}_{-\varepsilon}\left(V_{\bullet}\right)$.

Assumption. From now on we set $\varepsilon=-1$.

\subsection{Examples}

We consider three basic examples in types $C_{1}, C_{2}, \widetilde{C}_{1}$.

2.3.1 Type $C_{1}$. We take $N \geqslant 2, d_{0}=\ldots=d_{N / 2-1}=0, d_{N / 2}=d$. We have $V_{N / 2}=V=$ $\mathbb{C}^{d}, A_{N / 2}=A=A^{*} \in \operatorname{End}(V), B_{1}=0, p_{1}=p \in V, q_{1}=q \in V^{*}, q(v)=(p, v)$. Thus $\mathrm{M}_{d}^{\varepsilon}=\operatorname{End}^{+}(V) \oplus V$ and $\mathfrak{Z}_{d}^{\varepsilon}=\left(\operatorname{End}^{+}(V) \oplus V\right) / / O(V)$, where $\operatorname{End}^{+}(V) \subset \operatorname{End}(V)$ stands for the linear subspace of selfadjoint operators (symmetric matrices). By classical invariant theory, the ring of $O(V)$-invariant functions on $\mathrm{End}^{+}(V) \oplus V$ is freely generated by the functions $a_{1}, \ldots, a_{d}, b_{0}, \ldots, b_{d-1}$, where $a_{m}:=\operatorname{Tr}\left(A^{m}\right)$, and $b_{m}=\left(p, A^{m} p\right)$. Hence $\mathfrak{Z}_{\underline{d}}^{\varepsilon} \simeq \mathbb{A}^{2 d}$.

2.3.2 Type $C_{2}$. We take $N=4, d_{0}=0, d_{1}=d_{2}=d_{3}=1$. We have $V_{1}=V_{2}=V_{3}=\mathbb{C}$, and hence all our linear operators act between 1-dimensional vector spaces and can be written just as numbers. Hence $M_{d}^{\varepsilon}$ has coordinates $A_{1}=A_{3}, A_{2}, B_{1}=B_{2}, q_{2}=-p_{2}, q_{1}=p_{3}, q_{3}=p_{1}$, and $\mathrm{M}_{d}^{\varepsilon}$ is cut out by a single equation $B_{1}\left(A_{1}-A_{2}\right)=p_{2} p_{3}$. The group $G_{-\varepsilon}\left(V_{\bullet}\right)$ is the product $G L\left(V_{1}\right) \otimes O\left(V_{2}\right) \simeq \mathbb{C}^{*} \times\{ \pm 1\}$ with coordinates $c \in \mathbb{C}^{*}, s= \pm 1$. It acts on $M_{\underline{d}}^{\varepsilon}$ as follows:

$$
(c, s) \cdot\left(A_{1}, A_{2}, B_{1}, p_{1}, p_{2}, p_{3}\right)=\left(A_{1}, A_{2}, c s B_{1}, c^{-1} p_{1}, s p_{2}, c p_{3}\right) .
$$

The ring of $\mathbb{C}^{*} \times\{ \pm 1\}$-invariant functions on $\mathrm{M}_{\underline{d}}^{\varepsilon}$ is generated by the functions $A_{1}, A_{2}, b_{12}:=p_{2}^{2}$, $b_{01}:=p_{1} p_{3}, b_{02}:=p_{2} B_{1} p_{1}, b_{03}:=B_{1}^{2} p_{1}^{2}$ with three quadratic relations: $b_{02}\left(A_{1}-A_{2}\right)=b_{01} b_{12}$, $b_{03}\left(A_{1}-A_{2}\right)=b_{01} b_{02}, b_{02}^{2}=b_{12} b_{03}$. Thus $\mathfrak{Z}_{d}^{\varepsilon}$ is a 4-dimensional (noncomplete) intersection of three quadrics in $\mathbb{A}^{6}$. According to $\mathrm{S}$. Kovács (private communication) and computations by G. Leuschke and A. Verbitsky with the package Macaulay2, $\mathfrak{Z}_{\underline{d}}^{\varepsilon}$ is reduced, not $\mathbb{Q}$-Gorenstein, but is Cohen-Macaulay and normal, and has rational singularities.

2.3.3 Type $\widetilde{C}_{1}$. We take $N=2, d_{0}=d_{1}=1$. We have $V_{1}=\mathbb{C}=V_{2}$, and hence all our linear operators act between 1-dimensional vector spaces and can be written just as numbers. Hence $M_{\underline{d}}^{\varepsilon}$ has coordinates $A_{1}, A_{2}, B_{0}=B_{1}, q_{0}=p_{0}, q_{1}=-p_{1}$ and $\mathbf{M}_{\underline{d}}^{\varepsilon}$ is cut out by a single equation 


\section{Michael Finkelberg and LeOnid Rybnikov}

$B_{0}\left(A_{1}-A_{0}\right)+p_{1} q_{0}=0$. The group $G_{-\varepsilon}\left(V_{\bullet}\right)$ is the product $O\left(V_{0}\right) \times O\left(V_{1}\right) \simeq\{ \pm 1\} \times\{ \pm 1\}$ with coordinates $\left(s_{1}, s_{2}\right)$. The ring of $\{ \pm 1\} \times\{ \pm 1\}$-invariant functions on $\mathrm{M}_{\underline{d}}^{\varepsilon}$ is generated by the functions $A_{0}, A_{1}, b_{1}:=p_{1}^{2}, b_{0}:=p_{0}^{2}, s:=B_{0}^{2}$ with a single relation $b_{1} b_{0}-s\left(A_{0}-A_{1}\right)^{2}=0$. Note the coincidence with the output of [FR14, Example 2.8.3].

\subsection{The dimension of $\mathrm{M}_{\underline{d}}^{\varepsilon}$}

We define the factorization morphism $\Upsilon: M_{d}^{\varepsilon} \rightarrow \mathbb{A}^{\underline{d}}=\prod_{l \in I}\left(\mathbb{A}^{(1)}\right)^{\left(d_{l}\right)}$ so that the component $\Upsilon_{l}$ is just $\operatorname{Spec} A_{l}$.

Proposition 2.1. Every fiber of $\Upsilon$ has dimension $\operatorname{dim} G_{-\varepsilon}\left(V_{\bullet}\right)+\sum_{l \in I} d_{l}$.

Proof. We use the same argument as in the proof of [FR14, Prop. 2.11]. Let us list the minor changes necessary in the quadratic case. The dimension estimate for a general fiber is reduced to the dimension estimate for the zero fiber where all $A_{l}$ are nilpotent. By the adjointness condition, $\left(A_{\bullet}, B_{\bullet}, p_{\bullet}, q_{\bullet}\right)$ is determined by its components $A_{l}$ for $0 \leqslant l \leqslant N / 2, B_{l}$ for $0 \leqslant l<N / 2, p_{l}$ for $0<l \leqslant N / 2, q_{l}$ for $0 \leqslant l<N / 2$, and $A_{0} \in \operatorname{End}^{+}\left(V_{0}\right), A_{N / 2} \in \operatorname{End}^{+}\left(V_{N / 2}\right)$. Note that $\operatorname{dim} \operatorname{End}^{+}\left(V_{l}\right)=d_{l}\left(d_{l}+1\right) / 2$ for $l=0, N / 2$, while $\operatorname{dim} O\left(V_{l}\right)=d_{l}\left(d_{l}-1\right) / 2$ for $l=0, N / 2$. The dimension of the space of nilpotent selfadjoint operators in $\operatorname{End}^{+}\left(V_{l}\right)$ equals $d_{l}\left(d_{l}-1\right) / 2$ for $l=0, N / 2$. More generally, the space $\mathbb{O}_{\lambda}^{+} \subset \operatorname{End}^{+}\left(V_{l}\right)$ of nilpotent selfadjoint operators of Jordan type $\lambda$ (a partition of $d_{l}$ ) is a finite union of $O\left(V_{l}\right)$-orbits, all of the same dimension $\operatorname{dim} \mathbb{O}_{\lambda}^{+}=$ $\operatorname{dim} \mathbb{O}_{\lambda} / 2$ (where $\mathbb{O}_{\lambda}$ is the nilpotent $G L\left(V_{l}\right)$-orbit consisting of the nilpotent matrices of Jordan type $\lambda$ ), see [KR71, Prop. 5]. The argument of the proof of [FR14, Prop. 2.11] implies that, for example, the dimension of the space of $\left(A_{0}, A_{1}, B_{0}, p_{1}, q_{0}\right)$ subject to $A_{1} B_{0}-B_{0} A_{0}+p_{1} q_{0}=0$ is at most $d_{0}\left(d_{0}-1\right) / 2+d_{1}^{2}-d_{1}+\min \left(d_{0}, d_{1}\right)+\max \left(d_{0}, d_{1}\right)$. Summing up the similar estimates over $0 \leqslant l<N / 2$, we obtain the desired inequality $\operatorname{dim} \Upsilon^{-1}(0, \ldots, 0) \leqslant \operatorname{dim} G_{-\varepsilon}\left(V_{\bullet}\right)+\sum_{l \in I} d_{l}$. The opposite inequality follows from the computation of the generic fiber of $\Upsilon$, and the proposition follows.

The following corollary is proved the same way as [FR14, Cor. 2.12].

Corollary 2.2. The subscheme $\mathrm{M}_{\underline{d}}^{\varepsilon}$ is an irreducible reduced complete intersection in $M_{\underline{d}}^{\varepsilon}$.

THEOREM 2.3. The categorical quotient $\mathfrak{Z}_{\underline{d}}^{\varepsilon}$ is a reduced irreducible normal scheme.

Proof. We use the same argument as in the proof of [FR14, Thm. 2.7.a)]. We just list the minor changes necessary in the quadratic case. As in loc. cit., we have to check the normality of the open subscheme $U \subset \mathfrak{Z}_{\underline{d}}^{\varepsilon}$ defined as the preimage under the factorization morphism $\Phi: \mathfrak{Z}_{\underline{d}}^{\varepsilon} \rightarrow \mathbb{A} \underline{d}$ of an open subset $\hat{U} \subset \mathbb{A} \underline{d}$ formed by all the colored configurations where at most two points collide. As in loc. cit., this reduces to a few basic checks we already performed in the examples: 2.3.1 when two points of the same color 0 or $N / 2$ (outmost color for short) collide; [FR14, 2.8.1] when two points of the same color $l, 0<l<N / 2$ (innermost color for short) collide; 2.3 .2 when a point of an outmost color collides with a point of an inmost color; 2.3 .3 when two points of different outmost colors collide; [FR14, 2.8.2] when two points of different innermost colors collide. This completes the proof of the theorem.

\subsection{Symplectic zastava}

For $\bar{d}=\left(d_{0}, \ldots, d_{N / 2}\right) \in \mathbb{N}^{I}$ we consider the affine zastava space for the symplectic Lie group $G=S p_{N}$ introduced in [BFG06], where it is denoted by $\mathfrak{U}_{G ; B}^{\bar{d}}$. In the present paper we will 


\section{Quantization of Drinfeld Zastava in type C}

denote it by $Z_{\varepsilon}^{\bar{d}}$. It is a reduced irreducible affine scheme containing as an open subscheme the (smooth) moduli space $\stackrel{\circ}{Z}_{\varepsilon}^{\bar{d}}$ of degree $\bar{d}$ based maps from $\mathbb{P}^{1}$ to the affine flag scheme of $G=S p_{N}$.

Recall that $S p_{N}$ is the fixed point subgroup of the involutive pinning-preserving outer automorphism $\sigma: S L_{N} \rightarrow S L_{N}$. This automorphism acts on the affine flag scheme of $S L_{N}$, and on the zastava spaces $Z \underline{d}$ for $S L_{N}$. More precisely, we define $\underline{d}=\left(\widetilde{d}_{0}, \ldots, \widetilde{d}_{N-1}\right) \in \mathbb{N}^{N}$ as follows: for $0 \leqslant l \leqslant N / 2$ we have $\widetilde{d}_{l}=d_{l}$, and for $0<l<N / 2$ we set $\widetilde{d}_{N-l}=d_{l}$. Then $\sigma$ acts on $Z$, and the fixed point subscheme (with the reduced closed subscheme structure) is isomorphic to $Z_{\varepsilon}^{\bar{d}}$. In other words, $Z_{\varepsilon}^{\bar{d}}$ is the closure in $Z^{\underline{d}}$ of $\stackrel{\circ}{Z}_{\varepsilon}^{\bar{d}} \cong\left(\stackrel{\circ}{Z}^{\underline{d}}\right)^{\sigma}$.

Recall the chainsaw quiver variety $\mathfrak{Z}_{\underline{d}}$ introduced in [FR14]. Thm. [FR14, 2.7.b)] constructs a morphism $\eta: \mathfrak{Z}_{\underline{d}} \rightarrow Z^{\underline{d}}$, and [BF11, Thm. 3.5] proves that $\eta$ is an isomorphism. We will identify $\mathfrak{Z}_{\underline{d}}$ and $Z^{\underline{d}}$ via $\eta$. Under this identification the open subscheme $Z^{\underline{d}}$ corresponds to the open subscheme $\stackrel{\circ}{\mathfrak{Z}}_{\underline{d}}$ formed by the closed orbits of stable and costable quadruples $\left(A_{\bullet}, B_{\bullet}, p_{\bullet}, q_{\bullet}\right)$, that is, such that $V_{\bullet}$ is generated by the action of $A_{\bullet}, B_{\bullet}$ from the image of $p_{\bullet}$, and $V_{\bullet}$ contains no nonzero subspaces in $\operatorname{Ker} q_{\bullet}$ closed with respect to $A_{\bullet}, B_{\bullet}$. The fixed point subscheme $\left(\dot{\mathfrak{Z}}_{\underline{d}}\right)^{\sigma}$ coincides with $\stackrel{\circ}{\mathfrak{Z}}_{d}^{\varepsilon}$, the open subscheme of $\mathfrak{Z}_{d}^{\varepsilon}$ formed by the closed orbits of stable (equivalently, costable) quadruples $\left(A_{\bullet}, B_{\bullet}, p_{\bullet}, q_{\bullet}\right) \in \mathrm{M}_{\underline{d}}^{\varepsilon}, \underline{c f}$. [BS00, Table 1 and Prop. 3.3].

Lemma 2.4. The closed embedding $\stackrel{\circ}{\mathfrak{Z}}_{\underline{d}}^{\varepsilon} \cong\left(\stackrel{\circ}{\mathfrak{Z}}_{\underline{d}}\right)^{\sigma} \hookrightarrow \stackrel{\circ}{\mathfrak{Z}}_{\underline{d}}$ extends to the closed embedding $\mathfrak{Z}_{\underline{d}}^{\varepsilon} \hookrightarrow \mathfrak{Z}_{\underline{d}}$.

Proof. It suffices to check that any $G_{-\varepsilon}\left(V_{\bullet}\right)$-invariant function on $\mathrm{M}_{\underline{d}}^{\varepsilon}$ extends to a $\prod_{l=0}^{N-1} G L\left(V_{l}\right)$ invariant function on $\mathrm{M}_{\underline{d}}$. This is immediately seen on the generators provided by classical invariant theory.

Now, since $\stackrel{\circ}{\mathfrak{Z}}_{\underline{d}}^{\varepsilon}$ is dense in $\mathfrak{Z}_{\underline{d}}^{\varepsilon}=\left(\mathfrak{Z}_{\underline{d}}\right)^{\sigma}$, we conclude that $\mathfrak{Z}_{\underline{d}}^{\varepsilon} \subset \mathfrak{Z}_{\underline{d}}=Z^{\underline{d}}$ coincides with the closure of $\left(\grave{\mathfrak{Z}}_{\underline{d}}\right)^{\sigma}$ in $\mathfrak{Z}_{\underline{d}}=Z^{\underline{d}}$. Since the symplectic zastava scheme $Z_{\varepsilon}^{\bar{d}}$ also coincides with this closure, we arrive at the following result.

ThEOREM 2.5. There is a canonical isomorphism $\eta: \mathfrak{Z}_{d}^{\varepsilon} \stackrel{\sim}{\longrightarrow} Z_{\varepsilon}^{\bar{d}}$ making the following diagram commutative:

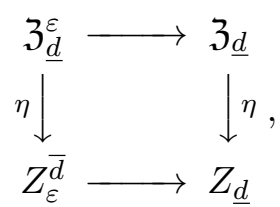

where the horizontal morphisms are the closed embeddings of the $\sigma$-fixed points subschemes.

COROLlary 2.6. The symplectic zastava scheme $Z_{\varepsilon}^{\bar{d}}$ is normal.

\section{Hamiltonian reduction}

\subsection{Poisson structures}

According to [FKMM99] (cf. [FR14, 3.1,3.3]), the smooth scheme $\stackrel{\circ}{Z}^{\underline{d}}$ carries a canonical symplectic structure which extends as a Poisson structure to $\mathfrak{Z}_{\underline{d}}=Z^{\underline{d}}$. This Poisson structure was constructed in [FR14] via Hamiltonian reduction. The restriction of the symplectic form on $Z^{\underline{d}}$ to $\stackrel{\circ}{Z}_{\varepsilon}^{\bar{d}} \cong\left(\stackrel{\circ}{Z}^{\underline{d}}\right)^{\sigma}$ coincides with the canonical symplectic form [FKMM99] on $\stackrel{\circ}{Z}_{\varepsilon}^{\bar{d}}$. We conclude that 


\section{Michael Finkelberg And LeONid Rybnikov}

the canonical symplectic structure on $\stackrel{\circ}{Z}_{\varepsilon}^{\bar{d}}$ extends as a Poisson structure to $Z_{\varepsilon}^{\bar{d}} \cong \mathfrak{Z}_{d}^{\varepsilon}$ and that the $\sigma$-fixed point embedding $\mathfrak{Z}_{\underline{d}}^{\varepsilon} \hookrightarrow \mathfrak{Z}_{\underline{d}}$ is Poisson. In the next subsection we will construct this Poisson structure on $\mathfrak{Z}_{\underline{d}}^{\varepsilon}$ via Hamiltonian reduction.

\subsection{Classical reduction}

Recall the Hamiltonian reduction definition of the Poisson bracket on zastava spaces in type A (see [FR14]). We "triangulate" the chainsaw quiver in the following way:

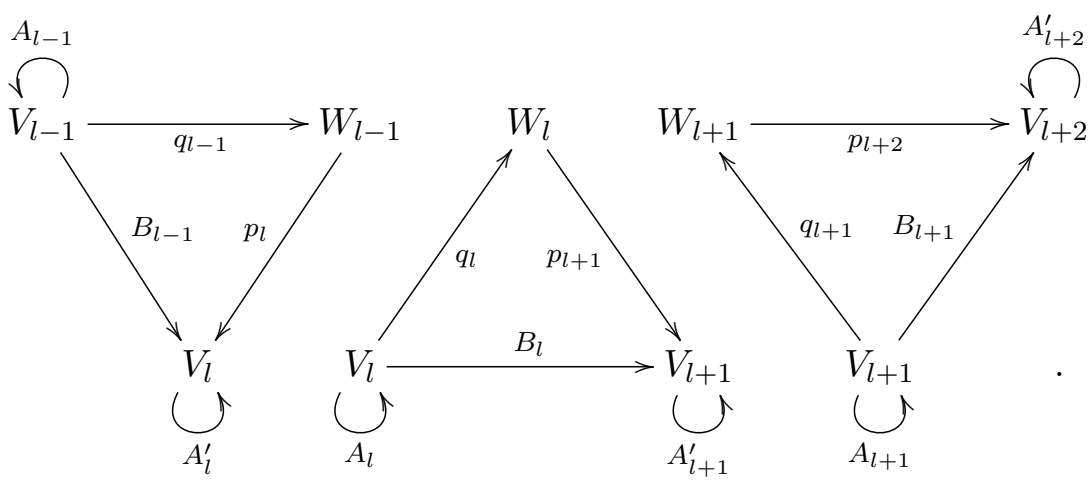

For a pair of vector spaces $U, V$ define the following 2-step nilpotent Lie algebra:

$$
\mathfrak{n}(U, V):=U \oplus V^{*} \oplus\left(U \otimes V^{*}\right),
$$

where the space $U \otimes V^{*}$ is central, $[U, U]=\left[V^{*}, V^{*}\right]=0$, and for $u \in U, v^{\vee} \in V^{*}$ one has $\left[u, v^{\vee}\right]=u \otimes v^{\vee}$.

To define the Poisson structure, we attach to each triangle of our graph the following Lie algebra:

$$
\mathfrak{a}_{l}:=\left(\mathfrak{g l}\left(V_{l}\right) \oplus \mathfrak{g l}\left(V_{l+1}\right)\right) \rtimes \mathfrak{n}\left(V_{l}, V_{l+1}\right)
$$

(the semidirect sum is with respect to the tautological action of $\mathfrak{g l}\left(V_{l}\right)$ on $V_{l}$ and $\mathfrak{g l}\left(V_{l+1}\right)$ on $\left.V_{l+1}^{*}\right)$.

Consider the Lie algebra

$$
\mathfrak{a}_{\underline{d}}:=\bigoplus_{l \in \mathbb{Z} / N \mathbb{Z}} \mathfrak{a}_{l}=\bigoplus_{l \in \mathbb{Z} / N \mathbb{Z}}\left(\mathfrak{g l}\left(V_{l}\right) \oplus \mathfrak{g l}\left(V_{l+1}\right)\right) \rtimes \mathfrak{n}\left(V_{l}, V_{l+1}\right) .
$$

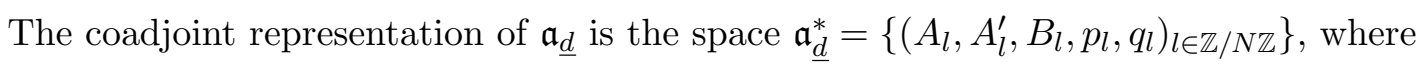

$$
A_{l} \in \operatorname{End}\left(V_{l}\right), \quad A_{l}^{\prime} \in \operatorname{End}\left(V_{l}\right), \quad B_{l} \in \operatorname{Hom}\left(V_{l}, V_{l+1}\right), \quad p_{l} \in V_{l}, \quad q_{l} \in V_{l}^{*} .
$$

Consider the subvariety $S_{\underline{d}} \subset \mathfrak{a}_{\underline{d}}^{*}$ defined by the following equations:

$$
B_{l} A_{l}+A_{l+1}^{\prime} B_{l}+p_{l+1} q_{l}=0, \quad l \in \mathbb{Z} / N \mathbb{Z} .
$$

Let $\mathfrak{g l}\left(V_{l}\right)_{\text {diag }}$ be the diagonal $\mathfrak{g l}\left(V_{l}\right)$ inside $\mathfrak{g l}\left(V_{l}\right) \oplus \mathfrak{g l}\left(V_{l}\right) \subset \mathfrak{a}_{\underline{d}}$ and let $\pi: \mathfrak{a}_{\underline{d}}^{*} \rightarrow \mathfrak{g l}\left(V_{l}\right)_{\text {diag }}^{*}$ be the projection. Then the Drinfeld zastava space $\mathfrak{Z}_{\underline{d}}$ is identified with the Hamiltonian reduction

$$
S_{\underline{d}} / / / \bigoplus_{l \in \mathbb{Z} / N \mathbb{Z}} \mathfrak{g l}\left(V_{l}\right)_{\mathrm{diag}}=\pi^{-1}(0) \cap S_{\underline{d}} / / \prod_{l \in \mathbb{Z} / N \mathbb{Z}} G L\left(V_{l}\right)_{\mathrm{diag}} .
$$

This provides a natural Poisson bracket on $\mathfrak{Z}_{\underline{d}}$.

The involution $\sigma$ acts on the space $\mathfrak{a}_{\underline{d}}^{*}$ as follows:

$$
A_{l} \mapsto-A_{N-l}^{*}, A_{l}^{\prime} \mapsto-A_{N-l}^{*}, B_{l} \mapsto B_{N-l}^{*}, p_{l} \mapsto q_{N-l}^{*}, q_{l} \mapsto p_{N-l}^{*} .
$$




\section{Quantization of DRinfeld Zastava in type C}

Remark 3.1. Strictly speaking, $\sigma$ is not an involution on $\mathfrak{a}_{\underline{d}}^{*}$ since $p^{* *}=-p$ and $q^{* *}=-q$, but becomes an involution after the Hamiltonian reduction.

To describe the fixed point set, we consider the half of the chainsaw quiver formed by the vertices $l \in I$. Define the Lie algebra

$$
\mathfrak{a}_{\underline{d}}^{\varepsilon}:=\bigoplus_{l=0}^{\frac{N}{2}-1} \mathfrak{a}_{l}=\bigoplus_{l=1}^{\frac{N}{2}-1}\left(\mathfrak{g l}\left(V_{l}\right) \oplus \mathfrak{g l}\left(V_{l+1}\right)\right) \rtimes \mathfrak{n}\left(V_{l}, V_{l+1}\right) .
$$

The coadjoint representation of $\mathfrak{a}_{\underline{d}}^{\varepsilon}$ is the space

$$
\mathfrak{a}_{\underline{d}}^{\mathcal{E}}=\left\{\left(A_{l}, A_{l}^{\prime}, B_{l}, p_{l}, q_{l}\right)_{l \in I_{0}}, A_{0}, q_{0}, B_{0}, A_{\frac{N}{2}}^{\prime}, p_{\frac{N}{2}}\right\},
$$

where

$$
A_{l} \in \operatorname{End}\left(V_{l}\right), \quad A_{l}^{\prime} \in \operatorname{End}\left(V_{l}\right), \quad B_{l} \in \operatorname{Hom}\left(V_{l}, V_{l+1}\right), \quad p_{l} \in V_{l}, \quad q_{l} \in V_{l}^{*} .
$$

The invariant subvariety $S_{\underline{d}}^{\varepsilon} \subset \mathfrak{a}_{\underline{d}}^{\varepsilon *}$ is again defined by the equations (1):

$$
B_{l} A_{l}+A_{l+1}^{\prime} B_{l}+p_{l+1} q_{l}=0, \quad l=0, \ldots, \frac{N}{2}-1 .
$$

Let $\mathfrak{o}\left(V_{l}\right) \subset \mathfrak{g l}\left(V_{l}\right) \subset \mathfrak{a}_{\underline{d}}^{\varepsilon}$ for $l \in I_{1}$ be the orthogonal Lie subalgebra and let

$$
\pi: \mathfrak{a}_{\underline{d}}^{\varepsilon *} \rightarrow \bigoplus_{l \in I_{0}} \mathfrak{g l}\left(V_{l}\right)_{\text {diag }}^{*} \oplus \bigoplus_{l \in I_{1}} \mathfrak{o}\left(V_{l}\right)^{*}
$$

be the projection. Then the symplectic Drinfeld zastava space $\mathfrak{Z}_{\underline{d}}^{\varepsilon}$ is identified (as a Poisson variety) with the Hamiltonian reduction

$$
S_{\underline{d}}^{\varepsilon} / / / \bigoplus_{l \in I_{0}} \mathfrak{g l}\left(V_{l}\right)_{\operatorname{diag}} \oplus \bigoplus_{l \in I_{1}} \mathfrak{o}\left(V_{l}\right)=\left(\pi^{-1}(0) \cap S_{\underline{d}}^{\varepsilon}\right) / / \prod_{l \in I_{0}} G L\left(V_{l}\right)_{\operatorname{diag}} \times \prod_{l \in I_{1}} O\left(V_{l}\right) .
$$

We denote the group $\prod_{l \in I_{0}} G L\left(V_{l}\right)_{\operatorname{diag}} \times \prod_{l \in I_{1}} O\left(V_{l}\right)$ simply by $G_{\underline{d}}$, and the corresponding Lie algebra $\bigoplus_{l \in I_{0}} \mathfrak{g l}\left(V_{l}\right)_{\operatorname{diag}} \oplus \bigoplus_{l \in I_{1}} \mathfrak{o}\left(V_{l}\right)$ by $\mathfrak{g}_{\underline{d}}$.

\subsection{Quantum reduction}

The natural quantization of the coordinate ring $\mathbb{C}\left[\mathfrak{a}_{d}^{\varepsilon *}\right]$ is the enveloping algebra $U\left(\mathfrak{a}_{d}^{\varepsilon}\right)$. It will be convenient to gather the generators of $U\left(\mathfrak{a}_{\underline{d}}^{\varepsilon}\right)$ (that is, the basis elements of the Lie algebra $\mathfrak{a}_{\underline{d}}^{\varepsilon}$ ) into the following $U\left(\mathfrak{a}_{\underline{d}}^{\varepsilon}\right)$-valued matrices:

$$
A_{k}, B_{k}, q_{k}, A_{l}^{\prime}, p_{l}, 0 \leqslant k<\frac{N}{2}, 0<l \leqslant \frac{N}{2} .
$$

According to [FR14], the coefficients of the following matrices form a subspace $R \subset U\left(\mathfrak{a}_{\underline{d}}^{\varepsilon}\right)$ invariant with respect to the adjoint action:

$$
B_{l} A_{l}+A_{l+1}^{\prime} B_{l}+p_{l+1} q_{l}, \quad l=0, \ldots, \frac{N}{2}-1, i=1, \ldots, d_{l+1}, j=1, \ldots, d_{l} .
$$

Equivalently, $U\left(\mathfrak{a}_{\underline{d}}^{\varepsilon}\right) R$ is a two-sided ideal in $\left.U\left(\mathfrak{a}_{\underline{d}}^{\varepsilon}\right)\right)$.

The natural quantization of the coordinate ring of the space $\mathfrak{Z}_{\underline{d}}^{\varepsilon}$ is the quantum Hamiltonian

reduction $y_{\underline{d}}^{\varepsilon}:=\left(U\left(\mathfrak{a}_{\underline{d}}^{\varepsilon}\right) / U\left(\mathfrak{a}_{\underline{d}}^{\varepsilon}\right)\left(R+\mathfrak{g}_{\underline{d}}\right)\right)^{G_{\underline{d}}}$. The ring $\underline{y}_{\underline{d}}^{\varepsilon}$ has a natural filtration coming from the PBW filtration on $U\left(\mathfrak{a}_{\underline{d}}^{\varepsilon}\right)$.

Proposition 3.2 (PBW property). We have gr $y_{\underline{d}}^{\varepsilon}=\mathbb{C}\left[\mathfrak{Z}_{\underline{d}}^{\varepsilon}\right]$. 


\section{Michael Finkelberg And LeONid Rybnikov}

Proof. The proof is a word for word repetition of that of Proposition 3.28 from [FR14].

We consider the following elements of $y_{\underline{d}}^{\varepsilon}$ :

$$
\begin{aligned}
& a_{l, r}:=\operatorname{Tr} A_{l}^{r}, r=1,2, \ldots, l \in I ; \\
& b_{l, s}:=q_{l} A_{l}^{s} p_{l}, s=0,1, \ldots, l \in I .
\end{aligned}
$$

We also introduce the following elements:

$$
\begin{gathered}
b_{k, l ; s_{k}, \ldots, s_{l}}:=q_{l} A_{l}^{s_{l}} B_{l-1} A_{l-1}^{s_{l-1}} B_{l-2} \ldots B_{k} A_{k}^{s_{k}} p_{k}, \quad k \leqslant l \in \mathbb{Z}, s_{i} \in \mathbb{Z}_{\geqslant 0} . \\
c_{k, l ; s_{k}, \ldots, s_{l}}:=B_{l} A_{l}^{s_{l}} B_{l-1} A_{l-1}^{s_{l-1}} B_{l-2} \ldots B_{k} A_{k}^{s_{k}}, \quad l=k+m N, s_{i} \in \mathbb{Z}_{\geqslant 0} .
\end{gathered}
$$

From the definitions we get the following relations.

Lemma 3.3. Let $k<l+1$. Then

$$
\left[b_{k, l ; 0, \ldots, 0}, b_{l+1,0}\right]= \begin{cases}b_{k, l+1 ; 0, \ldots, 0,0} & l \pm k \neq 0,2 \bmod N, 2 l+2 \neq 0 \quad \bmod N, \\ 2 b_{k, l+1 ; 0, \ldots, 0,0} & 2 l+2=0 \bmod N, \\ b_{k, l+1 ; 0, \ldots, 0,0}-b_{k-1, l ; 0, \ldots, 0,0} & l+k=0 \bmod N, \\ 2\left(b_{k, l+1 ; 0, \ldots, 0,0}-b_{k-1, l ; 0, \ldots, 0,0}\right) & l+k=0 \bmod N, 2 l+2=0 \\ b_{k, l+1 ; 0, \ldots, 0,0}-b_{k-1, l ; 0, \ldots, 0,0} & l-k=2 \bmod N,\end{cases}
$$

LEMma 3.4. For $k \leqslant m \leqslant l$, we have $\left[a_{m, r}, b_{k, l ; s_{k}, \ldots, s_{l}}\right]=\lambda b_{k, l ; s_{k}, \ldots, s_{m}+r-1 \ldots, s_{l}}+L$, where $\lambda \in$ $\mathbb{C} \backslash\{0\}, L \in y_{\underline{d}}^{\varepsilon}$ is expressed in $b_{k^{\prime}, l^{\prime} ; s_{k^{\prime}}, \ldots, s_{l^{\prime}}}$ with $l^{\prime}-k^{\prime}<l-k$ and $\operatorname{deg} L \leqslant \operatorname{deg} b_{k, l ; s_{k}, \ldots, s_{m}+r-1 \ldots, s_{l}}$ with respect to the $P B W$ filtration.

Proof. Straightforward.

Lemma 3.5. ( $C_{1}$ case) Let $p_{0}, p_{1}, A_{0}, A_{1}, B$ be the (matrices of) generators of the algebra $\mathfrak{a}_{d}$ for $N=2$. Then the algebra $y_{d}^{\varepsilon}$ is generated by $a_{l, r}:=\operatorname{Tr} A_{l}^{r}, b_{l, 0}:=p_{l}^{*} p_{l}$ with $l=0,1, r=1, \ldots, d_{l}$.

Proof. According to Lemma 3.4, it suffices to check that the invariants of the form $p_{0}^{*}\left(B^{*} B\right)^{m} p_{0}$, $p_{1}^{*} B\left(B^{*} B\right)^{m} p_{0}, p_{0}^{*}\left(B^{*} B\right)^{m} B^{*} p_{1}, p_{1}^{*}\left(B B^{*}\right)^{m} p_{0}$ and $\operatorname{Tr}\left(B B^{*}\right)^{m}$ can be expressed in $a_{l, r}, b_{l, 0}$. This is easily checked by induction on $m$.

Proposition 3.6. The algebra $y_{\underline{d}}^{\varepsilon}$ is generated by $a_{l, r}, b_{l, s}$ with $l \in I, r=1, \ldots, d_{l}$ and $s=0, \ldots, d_{l}-1$.

Proof. Arguing in the same way as in Proposition 3.35 of [FR14] we reduce the problem to expressing $b_{k, l ; 0,0, \ldots, 0}$ and $c_{0, m N ; 0, \ldots, 0}$ in $a_{l, r}, b_{l, s}$.

By Lemma 3.3 for $l-k<N-1$ we have $b_{k, l ; 0,0, \ldots, 0}=\lambda\left[\left[\ldots\left[b_{k, 0} b_{k+1,0}\right] \ldots, b_{l-1,0}\right], b_{l, 0}\right]$, where $\lambda$ is a nonzero number. Thus the $b_{k, l ; 0,0, \ldots, 0}$ with $l-k<n$ can be expressed in $a_{l, r}, b_{l, s}$. Suppose that $b_{k, k+m N-1 ; 0,0, \ldots, 0}$ for some $m \in \mathbb{Z}_{+}$can be expressed in $a_{l, r}, b_{l, s}$, then for $l-k<N$ we have $b_{k, l+m N ; 0,0, \ldots, 0}=\lambda\left[\left[\ldots\left[b_{k, k+m N-1 ; 0,0, \ldots, 0} b_{k+N+1,0}\right] \ldots, b_{l+N-1,0}\right], b_{l+N, 0}\right]$. Thus $b_{k, l+m N ; 0,0, \ldots, 0}$ with $l-k<N$ can be expressed in $a_{l, r}, b_{l, s}$ as well. So, the problem reduces to expressing $b_{k, k+m N-1 ; 0, \ldots, 0,0}$ and $c_{0, m N ; 0, \ldots, 0}$ in $a_{l, r}, b_{l, s}$.

Let $\underline{D}=\left(d_{0}, d_{\frac{N}{2}}\right)$. Define the homomorphism $\Phi: U\left(\mathfrak{a}_{\underline{D}}^{\varepsilon}\right) \rightarrow U\left(\mathfrak{a}_{\underline{d}}^{\varepsilon}\right)$ by

$$
\begin{gathered}
\Phi\left(A_{0}\right)=A_{0}, \Phi\left(A_{1}\right)=A_{\frac{N}{2}}, \Phi(B)=B_{\frac{N}{2}-1} \cdot B_{\frac{N}{2}-2} \cdot \ldots \cdot B_{0}, \\
\Phi\left(p_{0}\right)=p_{0}, \Phi\left(p_{1}\right)=B_{\frac{N}{2}-1} \cdot \ldots \cdot B_{1} p_{1} .
\end{gathered}
$$




\section{Quantization of Drinfeld Zastava in type C}

Note that $\Phi\left(y_{\underline{D}}^{\varepsilon}\right) \subset y_{\underline{d}}^{\varepsilon}$. By Lemma 3.5, $y_{\underline{D}}^{\varepsilon}$ is generated by the elements $a_{l, r}:=\operatorname{Tr} A_{l}^{r}$, $b_{l, 0}:=p_{l}^{*} p_{l}$ with $l=0,1, r=1, \ldots, d_{l}$. We have $\Phi\left(a_{0, r}\right)=a_{0, r}, \Phi\left(a_{1, r}\right)=a_{\frac{N}{2}, r}, \Phi\left(b_{0,0}\right)=b_{0,0}$, $\Phi\left(b_{1,0}\right)=b_{1, N-1 ; 0, \ldots, 0}$. Thus everything from $\Phi\left(\underset{D}{y_{D}^{\varepsilon}}\right)$ can be expressed in $a_{l, r}, b_{l, s}$. On the other hand, $\Phi\left(b_{0,2 m-1 ; 0, \ldots, 0}\right)=b_{0, m N-1 ; 0, \ldots, 0}$ and $\Phi\left(c_{0,2 m ; 0, \ldots, 0}\right)=c_{0, m N ; 0, \ldots, 0}$.

\section{Yangians}

\subsection{The Yangian of $\mathfrak{s p}_{N}$}

Let $\left(c_{k l}\right)_{k, l=1,2, \ldots, N / 2}$ stand for the symmetrized Cartan matrix of $\mathfrak{s p}_{N}$. That is, $c_{k k}=4$ for $k=N / 2, c_{k k}=2$ for $0<k<N / 2, c_{k l}=0$ for $|k-l|>1, c_{k l}=-1$ for $0<k, l<N / 2$ and $l=k \pm 1$, and $c_{k l}=-2$ otherwise.

The Yangian $Y\left(\mathfrak{s p}_{N}\right)$ is generated by $\mathbf{x}_{k, r}^{ \pm}, \mathbf{h}_{k, r}, k=1,2, \ldots, N / 2, r \in \mathbb{N}$, with the following relations:

$$
\begin{gathered}
{\left[\mathbf{h}_{k, r}, \mathbf{h}_{l, s}\right]=0,\left[\mathbf{h}_{k, 0}, \mathbf{x}_{l, s}^{ \pm}\right]= \pm c_{k l} \mathbf{x}_{l, s}^{ \pm},} \\
2\left[\mathbf{h}_{k, r+1}, \mathbf{x}_{l, s}^{ \pm}\right]-2\left[\mathbf{h}_{k, r}, \mathbf{x}_{l, s+1}^{ \pm}\right]= \pm c_{k l}\left(\mathbf{h}_{k, r} \mathbf{x}_{l, s}^{ \pm}+\mathbf{x}_{l, s}^{ \pm} \mathbf{h}_{k, r}\right), \\
{\left[\mathbf{x}_{k, r}^{+}, \mathbf{x}_{l, s}^{-}\right]=\delta_{k l} \mathbf{h}_{k, r+s},} \\
2\left[\mathbf{x}_{k, r+1}^{ \pm}, \mathbf{x}_{l, s}^{ \pm}\right]-2\left[\mathbf{x}_{k, r}^{ \pm}, \mathbf{x}_{l, s+1}^{ \pm}\right]= \pm c_{k l}\left(\mathbf{x}_{k, r}^{ \pm} \mathbf{x}_{l, s}^{ \pm}+\mathbf{x}_{l, s}^{ \pm} \mathbf{x}_{k, r}^{ \pm}\right), \\
{\left[\mathbf{x}_{k, r}^{ \pm},\left[\mathbf{x}_{k, p}^{ \pm}, \mathbf{x}_{l, s}^{ \pm}\right]\right]+\left[\mathbf{x}_{k, p}^{ \pm},\left[\mathbf{x}_{k, r}^{ \pm}, \mathbf{x}_{l, s}^{ \pm}\right]\right]=0, k=l \pm 1, k \in I, l \in I_{0}, \forall p, r, s \in \mathbb{N},} \\
\sum_{\sigma \in S_{3}}\left[\mathbf{x}_{k, r_{\sigma(3)}},\left[\mathbf{x}_{k, r_{\sigma(2)}},\left[\mathbf{x}_{k, r_{\sigma(1)}}, \mathbf{x}_{l, s}\right]\right]\right]=0, k=l \pm 1, k \in I, l \in I_{1}, \forall r_{1}, r_{2}, r_{3}, s \in \mathbb{N} .
\end{gathered}
$$

We will consider the "Borel subalgebra" $y^{\varepsilon}$ of the Yangian, generated by the $\mathbf{x}_{k, r}^{+}$and $\mathbf{h}_{k, r}$. For a formal variable $u$ we introduce the generating series $\mathbf{h}_{k}(u):=1+\sum_{r=0}^{\infty} \mathbf{h}_{k, r} u^{-r-1}$ and $\mathbf{x}_{k}^{+}(u):=\sum_{r=0}^{\infty} \mathbf{x}_{k, r}^{+} u^{-r-1}$.

We also consider a bigger algebra $\mathcal{D}^{\varepsilon}$, the "Borel subalgebra of the Yangian double", generated by all Fourier components of the series $\mathbf{h}_{k}(u):=1+\sum_{r=0}^{\infty} \mathbf{h}_{k, r} u^{-r-1}$ and $\mathbf{x}_{k}^{+}(u):=$ $\sum_{r=-\infty}^{\infty} \mathbf{x}_{k, r}^{+} u^{-r-1}$ (that is, the generating series $\mathbf{x}_{k}^{+}(u)$ are infinite in both positive and negative directions) with the defining relations $(5,6,8,9,10)$. The algebra $y \varepsilon$ is then the subalgebra generated by the negative Fourier components of the $\mathbf{x}_{k}^{+}(u)$ and $\mathbf{h}_{k}(u)$ due to the PBW property of the Yangians. We can then rewrite Eqs. $(6,8)$ in the following form:

$$
\begin{gathered}
\mathbf{h}_{k}(u) \mathbf{x}_{l}^{+}(v) \frac{2 u-2 v-c_{k l}}{2 u-2 v+c_{k l}}=\mathbf{x}_{l}^{+}(v) \mathbf{h}_{k}(u) . \\
\mathbf{x}_{k}^{+}(u) \mathbf{x}_{l}^{+}(v)\left(2 u-2 v-c_{k l}\right)=\left(2 u-2 v+c_{k l}\right) \mathbf{x}_{l}^{+}(v) \mathbf{x}_{k}^{+}(u) .
\end{gathered}
$$

The function $\left(2 u-2 v-c_{k l}\right) /\left(2 u-2 v+c_{k l}\right)$ here is understood as a formal power series in $u^{-1}, v^{-1}, u^{-1} v$, hence Eq. (11) is well defined. 


\section{Michael Finkelberg and LeOnid Rybnikov}

Following [FR14], we will use a little bit different generators of the Cartan subalgebra of the Yangian,

$$
\mathbf{A}_{k}(u):=u^{d_{k}}+A_{k, 0} u^{d_{k}-1}+\ldots+A_{k, r} u^{d_{k}-r-1}+\ldots
$$

obtained as the (unique) solution of the system of functional equations:

$$
\mathbf{h}_{k}(u)=\mathbf{A}_{k}\left(u+\frac{1}{2}\right)^{-1} \mathbf{A}_{k}\left(u-\frac{1}{2}\right)^{-1} \mathbf{A}_{k-1}(u) \mathbf{A}_{k+1}(u)\left(u+\frac{1}{2}\right)^{d_{k}}\left(u-\frac{1}{2}\right)^{d_{k}} u^{-d_{k-1}} u^{-d_{k+1}},
$$

for $k=1,2, \ldots, \frac{N}{2}-1$, and

$$
\begin{aligned}
\mathbf{h}_{\frac{N}{2}}(u)= & \mathbf{A}_{\frac{N}{2}}(u+1)^{-1} \mathbf{A}_{\frac{N}{2}}(u-1)^{-1} \mathbf{A}_{\frac{N}{2}-1}(u) \mathbf{A}_{\frac{N}{2}-1}\left(u+\frac{1}{2}\right) \\
& \cdot(u+1)^{\frac{d_{N}}{2}}(u-1)^{d_{\frac{N}{2}}} u^{-d_{\frac{N}{2}-1}}\left(u+\frac{1}{2}\right)^{-d_{\frac{N}{2}-1}} .
\end{aligned}
$$

Here we take $\mathbf{A}_{0}(u)=1$

Lemma 4.1. The generators $\mathbf{A}_{k}(u)$ of $\mathcal{D} y^{\varepsilon}$ satisfy the relations

$$
\mathbf{A}_{k}(u) \mathbf{x}_{l}^{+}(v) \frac{2 u-2 v+\frac{1}{2} c_{k k} \delta_{k l}}{2 u-2 v-\frac{1}{2} c_{k k} \delta_{k l}}=\mathbf{x}_{l}^{+}(v) \mathbf{A}_{k}(u) .
$$

Lemma 4.2. Let $\mathbf{A}_{k}(u)$ and $\mathbf{x}_{l}^{+}(u)$ be the generating series of $\mathcal{D} y \varepsilon$. Then the series

$$
\mathbf{a}_{k}(u)=\frac{\mathbf{A}_{k}\left(u-\frac{1}{4} c_{k k}\right)}{\mathbf{A}_{k}\left(u+\frac{1}{4} c_{k k}\right)}=1-d_{k} u^{-1}-\sum_{r=1}^{\infty} \mathbf{a}_{k, r} u^{-r-1} \quad \text { and } \quad \mathbf{x}_{l}^{+}(u)
$$

satisfy the following commutator relations:

$$
\left[\mathbf{a}_{k}(u), \mathbf{x}_{l}^{+}(v)\right](u-v)=-\frac{\frac{1}{4} c_{k k}^{2} \delta_{k l}}{u-v} \mathbf{x}_{l}^{+}(v) \mathbf{a}_{k}(u), \quad\left[\mathbf{a}_{k}(u), \mathbf{a}_{l}(v)\right]=0 .
$$

The series $\mathbf{a}_{k}(u), \mathbf{x}_{l}^{+}(u)$ generate $\mathcal{D}^{\varepsilon}$ with the defining relations (17), (8) and (9), and their negative Fourier components generate $y^{\varepsilon}$.

Proof. For $k \neq l$ the relation is obvious, for $k=l$ we have

$$
\mathbf{a}_{k}(u) \mathbf{x}_{k}^{+}(v) \frac{u-\frac{1}{4} c_{k k}-v+\frac{1}{4} c_{k k}}{u-\frac{1}{4} c_{k k}-v-\frac{1}{4} c_{k k}} \cdot \frac{u+\frac{1}{4} c_{k k}-v-\frac{1}{4} c_{k k}}{u+\frac{1}{4} c_{k k}-v+\frac{1}{4} c_{k k}}=\mathbf{x}_{k}^{+}(v) \mathbf{a}_{k}(u) .
$$

therefore

$$
\mathbf{a}_{k}(u) \mathbf{x}_{k}^{+}(v) \frac{(u-v)^{2}}{(u-v)^{2}-\frac{1}{4} c_{k k}^{2}}=\mathbf{x}_{k}^{+}(v) \mathbf{a}_{k}(u) .
$$

One can inductively express $\mathbf{A}_{k, r}$ in $\mathbf{a}_{k, s}$ with $s \leqslant r+1$, hence $\mathcal{D} y^{\varepsilon}$ is generated by $\mathbf{a}_{k}(u)$ and $\mathbf{x}_{l}^{+}(u)$. On the other hand, the quotient of $\mathbb{C}\left[\mathbf{a}_{k, r}\right]_{r=1}^{\infty} \cdot \mathcal{D} y^{+}$by relation $(17)$ is $\mathbb{C}\left[\mathbf{a}_{k, r}\right]_{r=1}^{\infty} \otimes \mathcal{D} y^{+}$ as a filtered vector space. The same argumentation holds for $y^{\varepsilon}$. The assertion follows.

\subsection{The Yangian of $\widehat{\mathfrak{s p}}_{N}$}

Let $\left(c_{k l}\right)_{k, l \in I}$ stand for the symmetrized Cartan matrix of $\widehat{\mathfrak{s p}}_{N}$. That is, $c_{k k}=4$ for $k=0$ or $k=N / 2, c_{k k}=2$ for $0<k<N / 2, c_{k l}=0$ for $|k-l|>1, c_{k l}=-1$ for $0<k, l<N / 2$ and $l=k \pm 1$ and $c_{k l}=-2$ otherwise. 


\section{Quantization of Drinfeld Zastava in type C}

As for the finite case, we will consider the "affine Borel Yangian". This is an associative algebra $\widehat{y} \varepsilon$ generated by the series

$$
\begin{gathered}
\mathbf{x}_{k}^{+}(u):==1+\sum_{r=0}^{\infty} \mathbf{x}_{k, r} u^{-r-1} \\
\mathbf{A}_{k}(u):=u^{d_{k}}+\sum_{r=0}^{\infty} \mathbf{A}_{k, r} u^{d_{k}-r-1}
\end{gathered}
$$

with $k \in \mathbb{Z}$, subject to the relations

$$
\begin{gathered}
\mathbf{A}_{k}(u) \mathbf{A}_{l}(v)=\mathbf{A}_{l}(v) \mathbf{A}_{k}(u), \\
\mathbf{x}_{k}^{ \pm}(u) \mathbf{x}_{l}^{ \pm}(v)\left(2 u-2 v \mp c_{k l}\right)=\mathbf{x}_{l}^{ \pm}(v) \mathbf{x}_{k}^{ \pm}(u)\left(2 u-2 v \pm c_{k l}\right),
\end{gathered}
$$

where $\left(c_{k l}\right)$ stands for the symmetrized Cartan matrix of $\widetilde{C_{n}}$, and

$$
\mathbf{A}_{k}(u) \mathbf{x}_{l}^{+}(v) \frac{2 u-2 v+\frac{1}{2} c_{k l} \delta_{k l}}{2 u-2 v-\frac{1}{2} c_{k l} \delta_{k l}}=\mathbf{x}_{l}^{+}(v) \mathbf{A}_{k}(u)
$$

in the sense that the negative Fourier components of the LHS and RHS are equal, and the Serre relations (9) and (10).

\subsection{Symplectic Yangians and symplectic zastava spaces}

THEOREM 4.3. The algebra $y_{d}^{\varepsilon}$ is a quotient of the Borel Yangian $\widehat{y} \varepsilon$ of $\widehat{\mathfrak{s p}}_{N}$ by some ideal containing $\mathbf{A}_{k, r}=0$ for $r>d_{k}$.

Proof. For $k \in I, l \in I_{0}$, we introduce the following generating series in $y_{\underline{d}}^{\varepsilon}$ :

$$
a_{k}(u):=1-d_{k} u^{-1}-\sum_{r=1}^{\infty} a_{k, r} u^{-r-1}, \quad b_{l}(u):=\sum_{s=0}^{\infty} b_{l, s} u^{-s-1} .
$$

For $l \in I_{1}, i=1,2, \ldots, d_{l}$, we introduce the following generating series in $U\left(\mathfrak{a}_{d}^{\varepsilon}\right)$ (warning: these are not in $\left.\underline{y}_{\underline{d}}^{\varepsilon}\right)$ :

$$
b_{l}^{(i)}(u):=\sum_{s=0}^{\infty} b_{l, s}^{(i)} u^{-s-1}, \quad b_{l, s}^{(i)}:=\left(A_{l}^{s} p_{l}\right)^{(i)},
$$

the $i$-th coordinate of the vector $A_{l}^{s} p_{l}$ in the orthonormal basis of $V_{l}$. We then have the following result (see [FR14]).

LEMma 4.4. The following relations hold:

$$
\begin{gathered}
(u-v)\left[b_{k}(u), b_{k}(v)\right]=\left(b_{k}(u) b_{k}(v)+b_{k}(v) b_{k}(u)\right) \text { for } k \in I_{0}, \\
2(u-v)\left[b_{k}(u), b_{l}\left(v+d_{l}\right)\right]=-\left(b_{k}(u) b_{l}\left(v+d_{l}\right)+b_{l}\left(v+d_{l}\right) b_{k}(u)\right) \text { for } k, l \in I_{0}, l=k+1, \\
(u-v)\left[a_{k}(u), b_{l}(v)\right]=-\frac{\delta_{k l}}{u-v} b_{l}(v) a_{k}(u) \text { for } k \in I, l \in I_{0} .
\end{gathered}
$$




\section{Michael Finkelberg and Leonid Rybnikov}

$$
\begin{gathered}
2(u-v)\left[b_{k}^{(i)}(u), b_{k}^{(j)}(v)\right]=c_{k l}\left(b_{k}^{(i)}(u) b_{k}^{(j)}(v)+b_{k}^{(j)}(v) b_{k}^{(i)}(u)\right) \text { for } k \in I_{1}, i, j=1, \ldots, d_{l}, \\
2(u-v)\left[b_{k}^{(i)}(u), b_{l}\left(v+d_{l}\right)\right]=c_{k l}\left(b_{k}^{(i)}(u) b_{l}\left(v+d_{l}\right)+b_{l}\left(v+d_{l}\right) b_{k}^{(i)}(u)\right) \\
\quad \text { for } k \in I_{1}, l=k+1, i=1, \ldots, d_{l}, \\
2(u-v)\left[b_{k}(u), b_{l}^{(i)}\left(v+d_{l}\right)\right]=c_{k l}\left(b_{k}(u) b_{l}^{(i)}\left(v+d_{l}\right)+b_{l}^{(i)}\left(v+d_{l}\right) b_{k}(u)\right) \\
\quad \text { for } l \in I_{1}, l=k+1, i=1, \ldots, d_{l}, \\
(u-v)\left[a_{k}(u), b_{l}^{(i)}(v)\right]=-\frac{\delta_{k l}}{u-v} b_{l}^{(i)}(v) a_{k}(u) \text { for } k \in I, l \in I_{1}, i=1, \ldots, d_{l} .
\end{gathered}
$$

Proof. This follows from Propositions 3.24 and 3.32 of [FR14].

LEMMA 4.5. We have

$$
\begin{gathered}
{\left[b_{k, r_{2}},\left[b_{k, r_{1}}, b_{l, s}\right]\right]+\left[b_{k, r_{1}},\left[b_{k, r_{2}}, b_{l, s}\right]\right]=0 \quad \text { for } k, l \in I_{0},|k-l|=1,} \\
{\left[b_{k, r_{2}},\left[b_{k, r_{1}}, b_{l, s}^{(i)}\right]\right]+\left[b_{k, r_{1}},\left[b_{k, r_{2}}, b_{l, s}^{(i)}\right]\right]=0 \quad \text { for } k \in I, l \in I_{1},|k-l|=1, i=1, \ldots, d_{l},} \\
{\left[b_{k, r_{2}}^{(i)},\left[b_{k, r_{1}}^{(j)}, b_{l, s}\right]\right]+\left[b_{k, r_{1}}^{(j)},\left[b_{k, r_{2}}^{(i)}, b_{l, s}\right]\right]=0 \quad \text { for } k \in I_{1}, l \in I,|k-l|=1 i, j=1, \ldots, d_{k} .}
\end{gathered}
$$

Proof. This follows from Proposition 3.32 of [FR14].

For $l \in I$, let $D_{l}(u)$ be the (unique) solution of the functional equation

$$
a_{l}(u):=D_{l}\left(u-\frac{1}{2}\right) D_{l}\left(u+\frac{1}{2}\right)^{-1} .
$$

We have

$$
D_{l}(u) b_{k}(v) \frac{2 u-2 v+\delta_{k l}}{2 u-2 v-\delta_{k l}}=b_{k}(v) D_{l}(u) \text { for } k \in I_{0}
$$

and

$$
D_{l}(u) b_{k}^{(i)}(v) \frac{2 u-2 v+\delta_{k l}}{2 u-2 v-\delta_{k l}}=b_{k}^{(i)}(v) D_{k}(u) \text { for } k \in I_{1} .
$$

Set $\widetilde{b_{k}^{(i)}}(u):=D_{k}\left(u-\frac{1}{2}\right)^{-1} b_{k}^{(i)}(u)$. From Lemma 4.4, we have

$$
\widetilde{b_{k}^{(i)}}(u) \widetilde{b_{k}^{(j)}}(v)=\widetilde{b_{k}^{(j)}}(v) \widetilde{b_{k}^{(i)}}(u) .
$$

Note that the rest of the relations for $b_{k}^{(i)}$ from Lemma 4.4 also hold for $\widetilde{b_{k}^{(i)}}$.

For $l \in I_{1}, i, j=1, \ldots, d_{l}$, set

$$
\widetilde{b_{l}^{(i j)}}(u):=D\left(u-\frac{1}{2}\right) \widetilde{b_{l}^{(i)}}(u) \widetilde{b_{l}^{(j)}}(u+1) .
$$

Note that

$$
\widetilde{b_{l}^{(i i)}}(u) \widetilde{b_{l}^{(j j)}}(v) \frac{u-v+2}{u-v-2}=\widetilde{b_{l}^{(j j)}}(v) \widetilde{b_{l}^{(i i)}}(u)
$$


and

$$
\widetilde{b_{l}^{(i i)}}(u) b_{k}(v) \frac{u-v+1}{u-v-1}=b_{k}(v) \widetilde{b_{l}^{(i i)}}(u)
$$

for $|k-l|=1$.

For $l \in I_{1}$, set $\widetilde{b_{l}}(u)=\sum_{i=1}^{d_{l}} \widetilde{b_{l}^{(i i)}}(u)$. By Proposition 3.6, we see that the algebra $y_{\underline{d}}^{\varepsilon}$ is generated by (the Fourier coefficients of) $D_{l}(u), b_{k}(u)$ for $k \in I_{0}$ and $\widetilde{b_{k}}(u)$ for $k \in I_{1}$. Now the theorem reduces to the following result.

Lemma 4.6. There is a homomorphism $\varphi_{\underline{d}}: \widehat{y} \varepsilon \rightarrow y_{\underline{d}}^{\varepsilon}$ sending $\mathbf{A}_{k}(u)$ to $D_{k}\left(u+\sum_{m=1}^{k} d_{m}\right)$ and $\mathbf{x}_{l}^{+}(u)$ to $b_{l}\left(u+\sum_{m=1}^{l} d_{m}\right)$ for $l \in I_{0}$ and to $\widetilde{b_{l}}\left(u+\sum_{m=1}^{l} d_{m}\right)$ for $i \in I_{1}$.

Proof. We need to prove the quadratic relations and the Serre relations for the elements $D_{l}(u)$, $b_{k}(u)$. The quadratic relations follow from the relations (37) and (38). The proof of the Serre relations is entirely similar to that of Proposition 3.32 from [FR14].

According to the Newton identity (see Theorem 7.1.3 of [Mol07]), we have

$$
a_{l}(u)=\frac{C_{l}\left(-u+d_{l}\right)}{C_{l}\left(-u+d_{l}-1\right)},
$$

where $C_{l}(u)$ is the Capelli determinant. This means that $D(u)=C\left(-u+d_{l}-\frac{1}{2}\right)$. In particular, $D_{l, r}=0$ for $r>d_{l}$.

Conjecture 4.7. $y_{\underline{d}}^{\varepsilon}=\widehat{y} \varepsilon /\left\{\mathbf{A}_{k, r} \mid r>d_{k}\right\}$.

\section{ACKNOWLEDGEMENTS}

We are grateful to D. Panyushev and D. Timashev for the help with references.

\section{REFERENCES}

BF11 A. Braverman and M. Finkelberg, Semi-infinite Schubert varieties and quantum K-theory of flag manifolds, J. Amer. Math. Soc., to appear, arXiv:1111.2266 (2011).

BFG06 A. Braverman, M. Finkelberg and D. Gaitsgory, Uhlenbeck spaces via affine Lie algebras, Progress in Math. 244 (2006), 17-135. http://dx.doi.org/10.1007/0-8176-4467-9_2

BS00 J. Bryan and M. Sanders, Instantons on $S^{4}$ and $\overline{C P}^{2}$, rank stabilization, and Bott periodicity, Topology 39 (2000), no. 2, 331-352. http://dx.doi.org/10.1016/S0040-9383(99) 00019-1

FKMM99 M. Finkelberg, A. Kuznetsov, N. Markarian and I. Mirković, A note on a symplectic structure on the space of G-monopoles, Commun. Math. Phys. 201 (1999), 411-421.

FR14 M. Finkelberg and L. Rybnikov, Quantization of Drinfeld Zastava in type A, Journal of Eur. Math. Soc. 16 (2014), 235-271.

GKLO05 A. Gerasimov, S. Kharchev, D. Lebedev and S. Oblezin, On a class of representations of the Yangian and moduli space of monopoles, Comm. Math. Phys. 260 (2005), 511-525. http: //dx.doi.org/10.1007/s00220-005-1417-3

Gua07 N. Guay, Affine Yangians and deformed double current algebras in type A, Advances in Math. 211 (2007), 436-484. http://dx.doi.org/10.1016/j.aim.2006.08.007

KP82 H. Kraft and C. Procesi, On the geometry of conjugacy classes in classical groups, Comment. Math. Helvetici 57 (1982), 539-602. http://dx.doi.org/10.1007/BF02565876 


\section{Michael Finkelberg And LeOnid Rybnikov}

KR71 B. Kostant and S. Rallis, Orbits and representations associated with symmetric spaces, Amer. J. Math. 93 (1971), 753-809.

KWWY12 J. Kamnitzer, B. Webster, A. Weekes and O. Yacobi, Yangians and quantizations of slices in the affine Grassmannian, arXiv:1209.0349 (2012).

Mol07 A. I. Molev, Yangians and classical Lie algebras, Math. Surveys and Monographs 143 AMS, Providence, RI (2007). http://dx.doi.org/10.1090/surv/143

Michael Finkelberg fnklberg@gmail.com

IMU, IITP, and National Research University Higher School of Economics, Department of Mathematics, 20 Myasnitskaya st, Moscow 101000, Russia

Leonid Rybnikov leo.rybnikov@gmail.com

IITP and National Research University Higher School of Economics, Department of Mathematics, 20 Myasnitskaya st, Moscow 101000, Russia 